AperTO - Archivio Istituzionale Open Access dell'Università di Torino

\title{
Polysynthese, Multifunktionalität und die denominalen Adjektive im Deutschen
}

\section{This is the author's manuscript}

Original Citation:

\section{Availability:}

This version is available http://hdl.handle.net/2318/133059

since

Publisher:

Walter de Gruyter

Published version:

DOI:10.1515/9783110228458

Terms of use:

Open Access

Anyone can freely access the full text of works made available as "Open Access". Works made available under a Creative Commons license can be used according to the terms and conditions of said license. Use of all other works requires consent of the right holder (author or publisher) if not exempted from copyright protection by the applicable law. 


\title{
Polysynthese, Multifunktionalität und die denominaler Adjektive im Deutschen*
}

\begin{abstract}
German is usually taken to have undergone a massive increase of analytic cons tions especially in inflection at the cost of earlier synthetic coding. However, we record the expansion of compounding, which is generally considered to be typic polysynthetic languages and is therefore in contrast with the general trend tov analyticity. This mixed picture, in which inflectional markers are eliminated but thetic features expand, leads to the paradox that the development of compoun also implies the increase of analyticity. In fact, in compounds modifiers typically their inflectional properties becoming invariable. This is also the case of verb mo ers like adverbs, predicative adjectives and nouns. Since this loss can be understor multifunctionality, which is a typical analytic property, the expansion of compoun turns out to imply the expansion of such multifunctionality, i.e. analyticity. Denon adjectives like Bombe, Klasse, etc., quite common among young people, reflect paradox.
\end{abstract}

\section{Zu einer typologischen Bewertung des Deutschen}

Ob die traditionellen Sprachtypen (fusionierend einschl. introflektierer agglutinierend - isolierend - polysynthetisch) reine theoretische Konst te darstellen oder doch auch eine gewisse Erklärungskraft bzw. prot penlogische Anziehungskapazität beanspruchen können, ist bis $h$ umstritten. Jedenfalls scheint es vernünftiger, die Typen als verschiec Kodierungstechniken aufzufassen. Das erlaubt es uns, das Potential unterschiedlichen Kodierungstechniken, das in den einzelnen Sprac vorhanden ist, darzustellen. Dies kann unter dem Schlagwort der Arb teilung subsumiert werden, und gibt uns ein Gesamtbild von Mischs chen, das fruchtbarer ist als eine scharfe Unterteilung nach grob defir ten Sprachtypen.

Wenn nun die diachrone Dimension ins Auge gefasst wird, kann komplexe Entwicklung eines historisch bestimmten Sprachsystems folgt werden. Es ist das Verdienst von Wurzel (1996a), die gesamte

Ich danke der Alexander-von-Humboldt-Stiftung für die Unterstützung meines Aufer tes in Berlin im Jahr 2009, der die hier vorgestellte Forschung ermöglichte. Auße möchte ich mich für hilfreiche Kommentare bei Ewald Lang bedanken. 
Man kann seine Ergebnisse folgendermaßen zusammenfassen: Die sionierende Technik ist zum großen Teil abgebaut. Allerdings mit ur schiedlichen Auswegen: Während bei den Verben fusionierende Ko rungen stark abgenommen haben und durch agglutinierende periphrastische/isolierende Konstrukte ersetzt bzw. um diese bereic wurden, ist bei den Nomina bzw. den Adjektiven eine Abnahme von sion nur teilweise zu beobachten. Zwar wird im heutigen Deutsch Kasusmarkierung durch die gesamte NP ausgedrückt, aber die Nume markierung ist viel resistenter und am Nomen sogar durch neu entstat ne introflektierende Kodierungen markiert (wie etwa den Umlaut in ter/Väter, u.ä.; vgl. dazu auch Primus 1997). Robuste Gegenbeispiel dieser Tendenz werden nur durch die derivationell offene Menge agentiven -er Suffigierungen geliefert. Darüber hinaus hat sich der fu nierende Charakter der Adjektivflexion wesentlich verstärkt. Das gilt a dings nur für den prototypischen Fall der attributiven Verwendung den anderen Verwendungen ist das Adjektiv einen entschiedenen Sc in die Richtung der isolierenden Kodierung gegangen. Wenn es a stimmt, dass durch Markierungsabbau Adverbien formal nicht mehr den Adjektiven zu unterscheiden sind, was zu einer für die isolieren Sprachen typischen diffusen Multifunktionalität führt, so hat sich aber Satzadverbien eine agglutinierende, spezifische Markierung entwic nämlich das Suffix -erweise. Schließlich ist eine eindeutig fusioniere Markierung im Bereich der Negation entstanden (vgl. kein, keiner, us eine wohl einmalige Erscheinung innerhalb der indoeuropäischen S chen.

Also: das Gesamtbild ist komplex. Einfach zu behaupten, dass Ar tik auf Kosten der Synthetik zugenommen hat, sagt wenig über die ty logische Charakterisierung des Deutschen (vgl. Roelcke 2004 für we Überlegungen). Was mir in Wurzels Übersicht besonders relevant scheint, ist die Beobachtung, dass die polysynthetischen Merkmale Lauf der deutschen Sprachgeschichte immer robuster geworden s Wenn im Althochdeutschen die Komposita wesentlich zweigliedrig wa so sind die formalen Möglichkeiten der Komposition, einschließlich Inkorporation, im heutigen Deutsch erheblich reichhaltiger. Das h dass sich diachron eine ganz neue Kodierungstechnik herausgebildet $b$ verstärkt hat, die den näher verwandten indoeuropäischen Sprachen großen Teil fremd ist, die aber Merkmale mit dem finno-ugrisc Sprachtyp teilt. Wie weit die jahrhundertelangen Sprachkontakte e 
Die Blüte der Polysynthese, so die in diesem Beitrag zu verteidige These, hat sich auf den gesamten deutschen Sprachtyp mehrfach nie geschlagen, da sich parallel zur Ausbreitung der Polysynthese das typ deutsche Muster der Klammerbildung entwickelt hat. Unter anderen ein Nebeneffekt der Klammerbildung die Ausbreitung der Multifunl nalität, was als ein typisches Merkmal der analytischen Sprachen gilt. Ausweitung der Komposition (synthetisches Merkmal) in Kombina mit der Klammerbildung führt dann im Deutschen zu einer Steigerung Multifunktionalität, d.h. der Analytik. Mit anderen Worten entsteht $p$ doxerweise aus der hohen Produktivität eines synthetischen Verfah eine Zunahme von analytischen Eigenschaften, die durch die Mech der Klammerbildung erzwungen werden. Das wird besonders im Fall aus Nomina entstandenen Adjektive deutlich.

Im Folgenden werden wir uns zuerst auf die Bedeutung der Poly these im Deutschen konzentrieren, dann werden wir das Thema Klam betrachten, das eng mit der Entwicklung der Polysynthese verbunden weil hier einerseits ein Konflikt zwischen Inkorporation und syntakt erzwungener Dislokation der Satzglieder entsteht. Andererseits prägt dadurch aber das spezifisch deutsche Muster der Inkorporation aus. diesem Hintergrund her werden wir das Hauptthema dieses Beitrags trachten, nämlich die Herausbildung der denominalen Adjektive 1 Prädikative, die in einigen Sprachregistern des heutigen Deutsch eine wisse Ausbreitung erfahren.

\section{Zur Bedeutung der Polysynthese im heutigen Deutsch}

Es muss eingangs angemerkt werden, dass die typologische Charakter rung der Polysynthese besonders problematisch ist. Bei Ineichen, der Charakteristische an diesem Sprachtyp in der „Gliederung aller gramn schen Funktionen mit Hilfe von Zusätzen um einen einzigen Stan sieht, ,so daß der Satz wie aus einem einzigen Wort bestehend ersche (1991: 50), liest man ganz deutlich (1991: 51): „„,Europäisch“ gese erscheint das Verfahren der Inkorporation besonders undurchsichtig schwierig. Als Beispiel dient gewöhnlich das Grönländische“. Dagege

43 Unabhängig von eventuellen Sprachkontakten ist aber sicherlich das polysynthet Potential der Komposition in allen germanischen Sprachen reichlich belegt, ebens spezifische Tendenz zur Entwicklung von inkorporierenden oder quasi-inkorporiere Mustern (vgl. Booij 2009 zum Niederländischen und Dahl 2004: 248-251 zu den nor manischen Sprachen). 
Sprachen überhaupt vertreten, ferner auch in etlichen finnisch-ugrisc Sprachen, so im Ungarischen, Finnischen, Lappischen. Seine hervor chendste Eigenschaft ist das reichliche Vorhandensein von Kompos Wie kommt man zu so unterschiedlichen Einschätzungen?

Es muss dazu gesagt werden, dass laut Skalička (1979: 57) „, [e]in w tiges Merkmal dieses Typs ist ..., daß die Wörter weder dekliniert $n$ konjugiert werden". Außerdem herrsche in diesem Typ freie Multifur onalität, da „Wörter einer bestimmten Bedeutung (Substantive, Verber der Bedeutung von formalen Wörtern, Präpositionen und Konjunktio verwendet werden“. Dementsprechend wird das Chinesische als mus haft polysynthetische Sprache betrachtet.

Da aber in medio stat virtus nicht nur ein schöner Spruch ist, können davon ausgehen, dass Komposition zwar von besonderer Bedeutung die Polysynthese ist, dies aber nicht direkt mit Multifunkionalität Affixlosigkeit korreliert. Also bleibt das Chinesische eine weitgehend lierende Sprache, wie man traditionell annimmt, und die Polysynthes dadurch charakterisiert, dass Komposition darin eine prominente $\mathrm{R}$ spielt. Insbesondere kann man als polysynthetisch diejenigen Sprac bezeichnen, die über produktive Inkorporationsmuster verfügen, w unter ,Inkorporation' Komposita mit verbalem Kopf verstanden wer (vgl. Aikhenvald 2007: 11). ${ }^{44}$ Ich lasse dahingestellt, ob solche Inkorp tions- bzw. Kompositionsmuster morphologischer oder syntaktis Natur sind. Mindestens für das Deutsche möchte ich aber dafür plädie dass sie auf ein bestimmtes Wortbildungsverfahren - sprich Morphol - zurückgeführt werden müssen (vgl. Gaeta im Druck).

Wenn wir nun die Komposition als Kennzeichen der Polysynt auffassen, erscheint es einschlägig, dass das Deutsche eine konsist Zunahme an Polysynthese erlebt hat. Im Althochdeutschen gab es e (1a) und unechte Komposita (1b), die meistens zweigliedrig waren.

44 Allerdings bedeutet das nicht, dass alle polysynthetischen Sprachen auch Inkorpor aufweisen müssen. Wie Iturrioz Leza (2001: 716) anmerkt, gibt es Sprachen, die zwar synthetisch sind, d.h. eine Anzahl von miteinander verknüpften lexikalischen oder matischen Morphemen aufweisen, aber keine Inkorporation haben. Womit auf die lichkeit referiert wird, „eine Anzahl von lexikalischen Morphemen zu einem komp Wort zu kombinieren“". Als Beispiel für eine nicht-inkorporiende, jedoch polysynthe Sprache sei auf Eskimo verwiesen, das, in der Regel ein lexikalisches Morphem pro hat", wie z.B. in angya-gblla-ng-yug-tuq ,Er will ein großes Boot kaufen, wrtl. BootERWERB-DESID-3SG'. Diese Unterscheidung erweckt jedenfalls die - hier nicht zu b wortende - Frage, in wie weit sich ein so definierter polysynthetischer Sprachtyp vor glutinierenden unterscheidet. 

c. $[$ bolz $[[$ werk $]$ [man $]]]$
,Holzarbeiter
d. [[hazal] [nul] kerno]
,Haselnusskern`

Längere Komposita sind seltener belegt. (1c-d) sind Beispiele für rechts- bzw. ein linksverzweigendes Kompositum. Außerdem gab schon Verbkomposita, aber nur mit Adjektiv als Erstglied gemäß folgenden drei Mustern:

(2) a. follabringan, vollbringen', follabuozen, Genüge tun', follagān ,beharren', follakweman ,gelangen“

b. missibrübhen ,missbrauchen', missibabèn ,sich fehlverhalten', missikwedan, Unrechtes reden', missitrüèn, misstrauen'

c. ebanbringan ,bringen', vgl. das lat. conferre, ebanbrübhen ,gebrauchen', lat. couti, ebandolen, ,Mitleid haben', lat. compati', ebanwirken ,mitwirken', lat. cooperar?

Verbkomposita mit nominalem Erstglied kommen im Althochdeutsc nur „mit alteriertem Verb“ (Henzen 1965: 92) vor:

(3) halswerfön ,den Hals drehen', muotbrehbōn ,sich verzehren', psalmosa ,lobsingen', rätslagōn ,ratschlagen', wunnisangōn ,jauchzen'

Man beachte, dass Komposita wie psalmosangön und wunnisangön als s nannte Parasyntheta gelten (vgl. Gaeta im Druck), da ihr Kopf $*_{s a}$ nicht als selbständiges Lexem vorkommt. In solchen Fällen scheinen Moment der Wortbildung Zusammensetzung und Ableitung zusamn gewirkt zu haben" (Henzen 1965: 238). ${ }^{45}$ Echte Verbkomposita mit minalem Erstglied sind erst im Mittelhochdeutschen zu finden (vgl. I zen 1965: 92):

(4) bönlachen ,hohnlachen', lügensträfen, verleumden', vederlesen ,federle zagelweiben, schwanzwedeln'

Schon Henzen weist allerdings darauf hin, dass solche Typen durch $\mathrm{R}$ bildung aus NN-Komposita entstanden sind. Man beachte, dass

45 Vielmehr können solche verbalen Komposita auf Nominalkomposita zurückgeführt den, die zum Teil auch belegt sind, wie bei psalmsang ,Psalter. Es ist klar, dass dieser tungsmechanismus der echten Rückbildung nahesteht (siehe unten im Text). 
stehung von Nominalkomposita mit verbalem Erstglied generell auf Reanalyse von echten NN-Komposita zurückgeführt, deren Erstglied deverbales Abstraktum war (vgl. Henzen 1965: 70):
a. decki-labhan
släf-hüs
stōz-isan
b. melkekubitin
skepfi-far.
werzi-stein
,Tuch zur Bedeckung
,Haus für den Schlaf
,Eisen für den Stoß`
,Melkeimer
,Schöpfgefäß`
,Wetzstein'

Aufgrund von real existierenden Abstrakta wie deckei, släf und stōz. vom Erstglied ein Verbalstamm decki-/släf-/stōz- rückgebildet und weitere Fälle ausgedehnt, in denen das Abstraktum nicht belegt war *melk. $/ *_{\text {skepfi }} / *_{\text {weral) }}$.

Es lässt sich also feststellen, dass alle Möglichkeiten bzw. Voraus zungen zur (weiteren Entwicklung der) Polysynthese schon in hochdeutscher Zeit vorhanden waren. Insbesondere sind in diesem sammenhang von Bedeutung: (a) die frei gegebene Möglichkeit Zusammensetzung von Lexemen, die mittels Analogie - d.h. von abst ten, konstruktionsmusterhaften Reanalyseprozessen - zu existieren Ableitungsmustern „polysynthetisch“ hergestellt werden, und (b) die fache Anwendung der damit verbundenen Rückbildung.

Was also im Althochdeutschen in nuce schon vorhanden war, das sich dann in der deutschen Sprachgeschichte „durch den schrittwe Abbau von strukturellen Restriktionen“ (vgl. Wurzel 1996a: 517) ma entwickelt. Insbesondere haben die durch Rückbildung bzw. durch Re lyse entstandenen inkorporierenden Verben zur vollen Blüte dieses K positionstyps mit verbalem Kopf geführt, der im heutigen Deutsch 1 unterschiedlichen Mustern produktiv ist:

- Reverbalisierungen von komplexen substantivierten Infinitiven: bausparen, probesingen;

- Reverbalisierungen von komplexen adjektivierten Perfektpartizipie platinbeschichten, schalldämpfen;

- Reverbalisierungen von deverbalen Nomina actionis: ehebrechen ( $<$ Ehebruch), notlanden ( $<$ Notlandung);

- Reverbalisierungen von Nomina agentis: beimwerken, testfahren. 


\section{Zur Klammer und zur Multifunktionalität}

Das Streben zur Reverbalisierung von komplexen Strukturen untersch lichen Ursprungs ist aber gleichzeitig eine nicht zu übersehende Quelle die Zunahme von diskontinuierlichen Sprachstrukturen im heut Deutsch. Als „diskontinuierender Sprachtyp“ (vgl. Werner 1979) weist Deutsche bekanntlich eine Reihe von klammerbildenden Entwicklur auf, die eindeutig eine Zunahme an Analytik darstellen (vgl. die Zus menstellung in Nübling 2006: 93):

- obligatorisches Subjektpronomen;

- Artikel;

- Präpositionen als Kasusersatz;

- grammatische Umschreibungen wie Perfekt, Plusquamperfekt, Fu Passiv, würde-Form;

- Modalverbkonstruktionen;

- Funktionsverbgefüge.

Ob sich aber das „klammernde Verfahren“ (Ronneberger-Sibold 19 qua Ziel bzw. qua Ergebnis einer Reihe von analytischen Sprachwan prozessen, auch als Zunahme von Analytik auffassen lässt, bleibe dahingestellt. ${ }^{46}$ Meiner Meinung nach lässt sich die so charakteristi Klammer-Bildung des Deutschen nicht unmittelbar auf den Wandel Synthetik zu Analytik beziehen, sondern eher auf allgemeine Entwick gen im Gestaltungssystem bzw. in der Linearisierung der Satzglied Sicherlich mag „der Übergang von der Kongruenz zur Diskontinu.

46 Vgl. zu dieser Frage Werner (1979: 981): „War es also eine zufällige Anhäufung von verschiedenen Ereignissen, die von der alten Kongruenz [d.h. Synthetik, LG] zur 1 Diskontinuität [d.h. Analytik, LG] geführt haben? Oder wirkte von Anfang an ein heimnisvolle Teleologie? Es war weder bloßer Zufall noch primäre Zielstrebigkeit, sor ein Wechselspiel zwischen gegebenen zufälligen Ereignissen und einer zweckvollen wahl, die aus der jeweiligen Situation etwas neu Funktionierendes gemacht hat, auch dies ein recht kompliziertes Prinzip zu sein scheint“. Diskontinuität ist ein solches Pr worunter „einer der Wege, wie sich aus vorhandenem Material neue Zeichen bilden la verstanden wird.

47 Vgl. ferner Admoni (1990: 2): „, [M]anche Gesetzmäßigkeiten, die sich z. B. in der Ent lung der deutschen Syntax geltend machen, sind durch den Hang zum analytischen nicht zu erklären. Dies gilt vor allem für die Wortstellung im deutschen Satz. Als Folg Flexionsreduzierung sollte man den Sieg der Kontaktstellung erwarten und den Übe zum Ausdruck der Beziehungen zwischen den Satzgliedern durch ihre Reihenfolge“. 


\section{Deutschen mitgewirkt haben.}

Um dem Risiko einer Henne-oder-Ei-Frage zu entgehen, können bei der Feststellung verbleiben, dass die Klammer ein wichtiges orgat torisches Prinzip des Deutschen ist. Diese vielleicht wohlfeile Anmerk hat aber eine Reihe von theoretischen und empirischen Konsequen die sogar den Begriff, Wort' im Deutschen in Frage stellen. Denn die nahme des Klammerverfahrens als normales und durchaus systematis und aktives Konstruktionsmuster impliziert, dass die syntaktische $T$ nung bzw. die Distanzstellung, die wir von den Partikelverben, aber a von den Verbkomposita kennen, keine Ausnahme, sondern die R darstellen. Insofern erscheinen die Versuche, eine einschlägige Tenc zur Univerbierung besonders bei Verbkomposita zu postulieren, als ab gig (vgl. diesbezüglich auch Wurzel 1998, und die kritische Auseinan setzung in Eisenberg 2004: 233-235). ${ }^{48}$

Es ist kein Zufall, dass Eschenlohr (1999) und Fuhrhop (2007) in taillierten Untersuchungen feststellen, dass die Mehrheit der Neubil gen mit ganz wenigen Ausnahmen eher regelmäßig trennbar bl Thurmair (1991) geht sogar davon aus, dass die Produktivität der Part verben ein entscheidender Faktor des klammernden Verfahrens ist. ${ }^{49}$

Somit ist auch theoretisch zu rechtfertigen, dass das Deutsche keinen „normalen“ Begriff, Wort' verfügt, sondern sich prinzipiell diskontinuierliche Strukturen spezialisiert hat (vgl. Thurmair 1991, W rich 2005: 29). Das muss wohl angenommen werden, wenn wir sov Partikelverben als auch Verbkomposita als „morphologisch-lexikalis Objekte ansehen wollen, denn die Trennbarkeit bzw. die Diskontin kann somit auf ein syntaktisches Prinzip zurückgeführt werden, das einer anderen Ebene auf das Wort als „zu Grunde liegende“ Einheit zug nimmt (vgl. Gaeta \& Ricca im Druck).

Wenn also die Trennbarkeit bzw. die Diskontinuität den Norma darstellt, aber zugleich polysynthetische Wortbildungsverfahren wie V

48 Diesbezüglich schlussfolgert Fuhrhop (2007: 59): „Insofern sind [die SubstantivVerbindungen] vom Prozess her das Gegenteil der Univerbierung: bei dieser wäch sammen, was häufig zusammensteht. Bei der Rückbildung ist das Besondere die Tren Es kann entsprechend auch als Gegenprozess zur Inkorporation gesehen werden: ei standteil eines Wortes wächst heraus und kann zum Teil Objektcharakter annehmen. vorher gerade kein Objekt".

49 Vgl. auch Feuillet (1989: 493) zur Ausbildung der Klammer bei den Partikelverben: position finale du préverbe „séparable“ ne s'est imposée d'une manière absolue que vement. Elle est le stade ultime du développement de l'allemand qui entraient en nexion étroite avec le verbe (complément directifs, compléments d'objet formant une préverbes). Ainsi se trouvait consacré l'ordre canonique du nha.". 
des flexionsmäßig aktiven Teils einer solchen komplexen Einheit vor setzt, ist die Folge, dass der übrigbleibende Teil flexionsmäßig inaktiv Das gilt für die Verbpartikeln, da Präpositionen im Normalfall (vgl. Nübling 2005 zu den ,artikulierten Präpositionen“) ohnehin nicht tierbar sind, d.h. über keine aktiven Flexionskategorien verfügen. Dass gilt aber auch für die Verbkomposita, wo das nominale Erstglied scherweise unflektiert bleibt (vgl. Fuhrhop 2007: 56):

(6) Karl spielt den ganzen Tag klavier/*klaviere.

Karl ist gestern bei der Rennprobe den ganzen Tag mehrmals autol *autos gefabren.

Außerdem hat sich der Grad der flexivischen Inaktivität auch bei den entstandenen komplexen Verbformen mindestens bezüglich der s nannten kontextuellen Flexion (vgl. Booij 1996) erhöht, indem im hochdeutschen vorkommende Flexionsmerkmale der Partizipien (forl a) und der Infinitive (geronn-e) aufgegeben wurden: ${ }^{50}$

(7) ioginuelich, thie thar gisibit unib sie zi geronne, in habet sia forlegana in si berzen

,derjenige, der eine Frau nur anschaut, um sie zur eigenen Lus gewinnen, hat sie schon in sein Herz verlegt ${ }^{6}$.

Insofern erscheint die Zunahme an flexivischer Inaktivität in allen von Thurmair (1991) angenommen Bereichen der Klammerbildung, $n$ lich in der lexikalischen, grammatischen und in der Kopulaklammer ein konvergierender Entwicklungsprozess, der in Richtung Analytik ge

Abbau von Flexionseigenschaften bedeutet offensichtlich Zunal an Analytik in dem Sinne, dass in den isolierenden Sprachen die WC flexionsmäßig vollkommen inaktiv bleiben, weil alle „flexivischen“ $\mathrm{M}$ male (im Extremfall auch Pluralität) mittels analytischer Konstruktio kodiert werden. Dagegen werden in den fusionierenden Sprachen nor lerweise die einzelnen Wörter durch explizite morphologische Ma charakterisiert, die gleichzeitig auch ihre Wortart fixieren. Diese le Eigenschaft ist eben den isolierenden Sprachen fremd. Insofern spiel Wortart im lexikalischen Sinne eine untergeordnete Rolle, was aber

50 Dass Flexionsabbau und Entwicklung von Periphrasen keine parallelen Prozesse müssen, zeigen die romanischen Sprachen, wo sich ähnliche Periphrasen entwickelt $h$ in denen aber die Flexionsmerkmale der nicht-finiten Verbteile nicht notwendiger verloren gegangen sind. 
Hier zeigt sich nun ein Paradox: durch die hohe Produktivität e polysynthetischen Verfahrens wie Komposition entsteht als Konsequ des typisch deutschen Merkmals der Klammerbildung eine einschlä Zunahme an Analytik, weil das nominale Erstglied eines Verbkomp tums flexivisch inaktiv wird. Wenn für ein Substantiv die flexivische I tivität merkwürdig erscheinen mag, so gilt das allerdings nicht für die nauso entstandenen unflektierten Adjektive, weil Adjektive im Deutsc bekanntlich nur positionsbezogen flexivische Aktivität aufweisen. Da typologisch gesehen eine hochinteressante diachrone Entwicklung: In prototypischen, attributiven Verwendung hat sich die Flexionsvie sogar vergrößert, und zwar mit einer Umgestaltung, die das Merk [ \pm Definitheit] funktionalisiert bzw. grammatikalisiert hat (vgl. Lockw 1968: 41-42). Andererseits ist in der prädikativen Verwendung die $\mathrm{F}$ tierbarkeit immer mehr abgebaut worden. Im Althochdeutschen war Flexion noch vorhanden, aber schon schwankend (vgl. (8a-b)) und re schnell, d.h. im Frühneuhochdeutschen, praktisch abgebaut (vgl. L wood 1968: 40):

(8) a. sie sint ... wisduames folle

,sie sind voll der Weisheit ${ }^{\star}$

b. thie ziti sint sō heilag

,die Zeiten sind so heilig'

Das unterschiedliche Schicksal des Flexionsverhaltens bei attributiver prädikativer Verwendung (Richtung Synthetik im ersten Fall und Ana im zweiten) ist besonders auffällig und meines Wissens noch nicht ric begriffen worden (vgl. allerdings Vogel 1997). Darüber hinaus wird c divergierende Entwicklung von der kategorialen Neutralisierung der malen Kodierung begleitet, indem der Adverbmarker -o (vgl. ahd. stark starko usw.) genau in der gleichen Zeitspanne abgebaut wurde. Dass c Entwicklung nicht notwendigerweise das Ergebnis einer rein phonol schen Schwächung und dann Tilgung des Markers darstellen muss, das Englische, wo sich in Anwesenheit einer ähnlichen phonologisc Reduzierung eine neue (agglutinierende!) Kodierung für Adverbien ausgebildet hat, nämlich das Suffix - $-y$. Trotz der in den beiden eng wandten Sprachen nachweisbaren massiven Produktivität dieses Suff das sogar an schon existierende Adjektive angehängt wurde, wod neue Adverbien entstanden (z. B.: wär > wärlih > wärlïhho, vgl. Lockw 1968: 51), und trotz der Spuren dieser adverbialen Funktion, die noch solchen Bildungen bestehen bleiben, indem z. B. schwerlich, wahrlich, fü neulich, bitterlich nur als Adverbien verwendet werden können (vgl. Her 1965: 230), haben sich Englisch und Deutsch in entgegengesetzte R 
krassem Widerspruch zur generellen Entwicklung der beiden Sprac steht.

Die oben skizzierte Entwicklung der Klammerbildung, in der ei flektierten Glied ein unflektiertes Glied in Distanzstellung gegenüberst liefert vielleicht einen Hinweis zur Erklärung dieser Reihe von ansc nend widersprüchlichen Entwicklungen, nämlich Flexionsabbau bei pr kativen Adjektiven gegenüber Flexionsausbau bei den attributiven, Abbau der Adverbmarker im Deutschen gegenüber Grammatikalisier eines Adverbmarkers im stark zur Analytik neigenden Englischen. sächlich erscheint ein bedeutsamer Parallelismus zu bestehen zwischen Klammerbildung bei Verbkomposita und Partikelverben und der Dislc rung von prädikativ bzw. adverbial gebrauchten Adjektiven.

Sicherlich kann die Flexionsinaktivität, die sich bei prädikativ bzw. verbial gebrauchten Adjektiven und Verbkomposita herausgebildet sprachtypologisch auf einen gemeinsamen Nenner zurückgeführt wer Diese Fälle von Analytikzunahme schlagen sich in der deutschen Gr matik als Verwischung der Wortartengrenzen nieder, da der Verlus Flexion auch die Verdunklung der Wortartzugehörigkeit - d.h. die M funktionalität (vgl. Vogel 1996: 231) - einschließt. Als Nebeneffekt dann die Klammerbildung von Multifunktionalität begleitet. Obwohl Klammerbildung nicht einfach als analytisches Verfahren aufgefasst den kann, trägt sie zu deutlicher Zunahme von isolierenden Merkm und insbesondere zur Multifunktionalität bei. Es sei hervorgehoben, Multifunktionalität bzw. flexivische Inaktivität und Distanzstellung sov in der prädikativen als auch in der adverbialen Funktion bzw. bei V komposita und Partikelverben zu beobachten sind.

\section{Die denominalen Adjektive}

Auf dem Hintergrund dieser Überlegungen ist nun eine kleine Gru von Nomina zu untersuchen, die augenfällig als Adjektive verwendet den können. Traditionell sind sie unter dem Etikett von Konversio erfasst. Henzen (1965: 247) listet unter Konversion bzw. „Klassenwec von Wörtern in ihrer Normalform“ eine erhebliche Menge von Fällen

51 Vgl. Lockwood (1968: 52): „The situation in this respect in OE was quite comparal OHG, but the analogous possibilities were developed in reverse proportions. For wh in modern German the uninflected adjective can always be an adverb and formatic suffix is rare, in English the latter is now virtually universal and the former very muc stricted". 
as. ahd. harm, ahd. durft, giwar, giwon, zweinzug - zwanzig usw., mhd. (ahd. fruma Nutzen), ernst, gewalt, schult, schade, angst, wette, wēnec, schach (und) mat, vīe)nt feind (gesteigert vīnder, vīndest), nōt (Kompar. nơ nhd. brach, fehl, schmuck, schnuppe, wurst, wrac (nd.), ekel, esel (vil esler pa DWb. 3, 1148), abrede, bank(e)rott, rosa, lila, u. ä.

Trotzdem scheint vielen, wenn nicht allen, Forschern der Konversions tus von solchen denominalen Adjektiven fraglich zu sein. Die radik Stellungnahme kann man Motsch (2004: 179) entnehmen, der „ke Grund [sieht], diese innerlexikalische Beziehung als ein Wortbildungs ster zu beschreiben". Sonst sprechen die meisten Forscher von Kon sion bzw. Konvertaten, obwohl eingeräumt wird, „dass man statt e Wortbildungsprozesses bei streng synchroner Betrachtung auch kategc le Mehrfachmarkierung eines Lexems bzw. eines Stammes anneh kann" (Altmann \& Kemmerling 2005: 150). Es ist auch nicht klar, solche Wortbildungsprodukte überhaupt als Adjektive angesehen we sollen“" (Donalies 2002: 133). Man beachte allerdings, dass Donalies ih trotz ihrer unsicheren Wortartzugehörigkeit den Status von Wortbildu. produkten zubilligt.

In diesem Zusammenhang möchte ich Motsch zustimmen und Begriff Konversion - mindestens im morphologischen bzw. syntaktisc Sinne - für andere Wortbildungsmuster reservieren. ${ }^{52}$ Unter morphol scher Konversion verstehen wir die klassischen Fälle von nominaler $\mathrm{k}$ verbaler Wortbildung wie Ruf, Scblag bzw. buttern, ölen (vgl. Eschen 1999: 46). Darüber hinaus verstehen wir unter syntaktischer Konver bzw. Transposition solche Fälle, in denen ein Lexem als Kopf einer minalen Phrase verwendet wird wie das Schöne bzw. das Begreifen. Sc bekommt es gewisse morphologische Eigenschaften, beispielsweise n rales Genus, andere morphologische Eigenschaften bleiben ihm aber schlossen, beispielsweise Pluralisierbarkeit. Das Hauptkennzeichen syntaktischer Konversion ist eine uneingeschränkte Produktivität, dic von der morphologischen Konversion unterscheidet. Nicht jedes Nor kann Kopf einer verbalen Phrase werden, während jeder Infinitiv Kopf einer nominalen Phrase gemacht werden kann. Uneingeschrä Produktivität scheint eher flexionsmorphologischer bzw. syntaktische derivationsmorphologischer Natur zu sein (vgl. Wurzel 1996b, G 2007, 2009).

52 Allerdings scheint es auch fraglich zu sein, ob man bei der syntaktischen Konve wirklich von einem Wortbildungsmuster sprechen sollte, weil dadurch kaum ein $n$ wenn auch nur potentiell lexikalisierbares Lexem zustande kommt (vgl. Hohenhaus 20 
uneingeschränkt produktiv sind, obwohl darauf hingewiesen werden $\mathrm{m}$ dass ihnen ein beschränktes Expansionspotential mindestens fachspr lich zuerkannt werden muss (siehe unten).

Andererseits sind die denominalen Adjektive auch nicht als morn logische Konversionen zu betrachten, weil ihr morphologisches Verha untypisch ist. Nur in Einzelfällen können sie beispielsweise attributiv, dann begrenzt flektiert, verwendet werden. Man beachte, dass die attr tive Funktion als prototypisch für die Adjektiva überhaupt angenom wird. Insofern bilden die denominalen Adjektiva eine ganz untypi Gruppe. ${ }^{53}$ Wenn man darüber hinaus unter Produktivität eines Wor dungsverfahrens die Gesamtmenge der Einheiten versteht, auf die Verfahren anwendbar ist, dann kann man den denominalen Adjekt derivationsmorphologische Produktivität absprechen, weil keine of sichtlichen Beschränkungen vorliegen.

Wenn sich nun der morphologische Weg als keine gängige Erklär erweist, stellt sich die Frage nach der Entstehung dieser kleinen Gru In diesem Zusammenhang scheint mir Eichinger voll zuzustimmer sein, wenn er (2007: 176) beobachtet, dass „es die Verwendung als Ac pula [ist], also im prädikativen oder zumindest im nichtnominalen $\mathrm{K}$ text, die den Weg vom Substantiv zum Adjektiv eröffnet“. Mit and Worten: die Verwendung dieser Nomina in prädikativer Funktion, Multifunktionalität bzw. Dekategorialisierung herrscht, ist dafür ver wortlich, dass eben diese Nomina als „Eigenschaftswörter" umzukatę sieren sind.

Allerdings ist fraglich, ob auch seiner Behauptung zuzustimmen dass der im Vergleich mit der substantivischen Verwendung von Ac tiven weitaus weniger systematische Charakter dieser Art von Umk gorisierung damit zu tun habe, „dass an dieser Stelle die Möglichke der desubstantivischen Derivation den wesentlichen Teil der Transp tionsarbeit leisten, wobei durch die Suffixe auch Probleme des morph gischen Anschlusses vermieden werden" (Eichinger 2007: 176). D Vermutung erklärt nämlich nicht, wieso die durchaus produktive Subs tivierung von Adjektiven nicht von den hochproduktiven nominalen fixen wie -heit (und Allomorphen) bzw. -ität beeinträchtigt wird, vgl. tematische Paare wie das Schöne/die Schönheit bzw. das Produktiv Produktivität usw.

53 Es sei am Rande angemerkt, dass bei den suffigierten denominalen Adjektiven gerad Gegenteil zu beobachten ist: sie können normalerweise in attributiver Funktion verw werden, aber nur in beschränktem Maß können sie auch prädikativ bzw. adverbial sein 
denominalen Adjektive mit einer solchen Erklärung erfassen lassen. W rend ein Fall wie feind durchaus über eine prädikative Verwendung erl bar ist, scheint dieselbe Erklärung für klasse unangemessen, wie den genden Google-Beispielen zu entnehmen ist: ${ }^{54}$

(9) a. Obgleich kein Feind mir feinder ist als jener lose Knabe, Gott weiss es, ich lieber ihn als meine Seele babe.

b. deine Torte ist mir Klasse gelungen.

c. Vor allem die Musik gefiel mir klasse.

Man beachte einerseits, dass feind sogar eine Komparativform zulässt, andererseits, dass klasse vornehmlich in adverbialer Funktion vorkon die eben feind fremd ist. Außerdem weist feind eine klare Argumentstru auf, wo ein Wahrnehmender im Dativ als Komplement kodiert ist, was klasse ungrammatisch ist:

(10) a. Sie ist mir feind.

b. * Sie ist mir klasse.

Andererseits ist klasse in adverbialer Funktion üblich (vgl. (9b-c)), die feind unmöglich ist, was nur schlecht mit einer strikten adkopulativen wendung zusammenpasst.

Daher scheint mir der Terminus ,Adkopula' generell unglücklich sein. Mit diesem Begriff bezeichnen Zifonun, Hoffmann \& Stre (1997: 55) diejenigen Komplemente einer Kopula, die „auf diese Funk spezialisiert“ sind, d.h. eine Kategorie von Wörtern, die „also - ander die auch in dieser Funktion vorkommenden Adjektive - nicht attrib verwendet werden [können] und nicht flektierbar [sind]". Sie sollen „Grundausdruck der Kategorie Prädikativ“ bilden (vgl. Zifonun, H mann \& Strecker 1997: 979):

Prädikative rekrutieren sich aus folgenden Ausdrucksklassen (z.T. durch Um gorisierung): aus der Kategorie der Adkopula - diese (wie z.B. quitt, gewillt, sind Grundausdrücke und erhalten somit direkt die Kategorie PRD; aus der tegorie der Adjektivphrasen; aus der Kategorie der Nomina (Hans ist Bäcker).

54 Außerdem machte mich Ewald Lang darauf aufmerksam, dass streng genommen s denominalen Adjektive nur analytische Komparative bzw. Superlative bilden: Er ist mi feind als $d u / v o n$ allen am meisten feind. In dem Beispiel soll die Vorerwähnung des Subst Feind die Bildung eines synthetischen Komparativs begünstigt haben. 
klasse definiert werden kann. Elemente dieser Klasse sind nämlich solche Lexeme - meistens verschiedener lexikalischer Natur, wie die angeführten Beispiele, die eben denominalen, departizipialen bzw. fr den Ursprung haben -, die nur in der prädikativen Funktion vorkomn Es ist damit aber auch impliziert, dass ein Lexem, sobald es auch in a butiver Funktion vorkommt, seine adkopulative Mitgliedschaft einbüß

Trotz meiner persönlichen Präferenz für eine positive Aufwertung in einer Sprache nachweisbaren Wortarten scheint mir der Begriff Ac pula bestenfalls eine Umbenennung der prädikativen Funktion zu wobei allerdings zwischen syntaktischer Funktion und Wortart ur schieden werden muss (vgl. oben zum Chinesischen und Vogel 1996: 230). In diesem Sinne kann man sicherlich von einer adkopulativen prädikativen Funktion sprechen. Die Postulierung einer eigenen Wortl se, die allerdings im Unterschied zu Adjektiven und Nomina nicht $\mathrm{d}$ Wortbildungsmuster erweitert werden kann, scheint mir aber eine w nützliche Verkomplizierung der Darstellung.

Wenn wir uns nun wiederum die kleine Gruppe der denominalen jektive ansehen, können wir mindestens drei Typen feststellen. Den er Typ, in dem die Argumentstruktur eines typischen psychischen Verbs einem obliquen Wahrnehmenden vorkommt (vgl. Wegener 1999: 1 haben wir schon erwähnt:

\section{angst, bange, ekel, elend, ernst, feind, freund, leid, panne, recht, schade, schuld}

Man beachte, dass dieser Typ ziemlich alt ist, mit Beispielen, die in mittelhochdeutsche Zeit zurückreichen. Zum Teil weisen die älteren spiele andere Adjektiveigenschaften wie zum Beispiel Komparation (vgl. feind, aber auch esel). Außerdem lässt sich dieser Typ nicht nur Kopula (sein, werden, bleiben) bzw. einem anderen Funktionsverb (tun) bachten, sondern auch als reines Verbprädikativ mit anderen kausat Prädikaten und schon in älterer Zeit, wie in Es macht dir angst und bange.

55 Zum Beispiel wird auch fit als Adkopula aufgefasst (vgl. Zifonun, Hoffmann \& Str 1997: 986): sobald aber fit auch in attributiver Funktion verwendet wird, hört es offen lich auf, eine Adkopula zu sein, und wird zum normalen Adjektiv. Beispiele aus Goog. habe das erst zweimal probiert, denn ich möchte ja nicht als Gangsta enden, sondern ein fitter Jung ben. Oder sollen wir hier von einer ,deadkopulativen Konversion' sprechen?

56 Das ist ein weiterer Grund, weshalb der Begriff Adkopula unangemessen erschein etwa auch machen als Kopula aufgefasst werden? 
Typs eine prädikative Einheit mit der Kopula bilden, und andere St prädikate selegieren können, steht nicht unerwarteterweise in Überein mit der gesamten Klammer-Bildung dieser Konstruktion:

(12) a. *Es ist mir feind sicherlich gewesen.

b. Es ist mir sicherlich feind gewesen.

Der zweite Typ ist auch alt und besteht aus Massennomina:

(13) kacke, käse, mist, müll, sahne, scheiße, schmuck, schrott, schnuppe, wurst/ wurscht

Bei diesen Wörtern findet über die Prädikation die Zuschreibung gewissen positiven bzw. negativen Bewertung statt, die konnotativ dem Massennomen assoziiert ist. Die Konnotation ist übrigens ein vanter semantischer Bestandteil einer solchen askriptiven Prädikation Pittner \& Berman 2006). Als Untertyp der askriptiven Prädikation gilt Variante mit Artikel, die bei Nomina, die zählbar sind, vorkommt. kann entweder direkt bei pluralisierbaren Nomina durch einen Grin Prozess entstehen, die aber auch ohne Artikel als Massennomina au fasst werden können (vgl. (14a) unten mit Google-Beispielen); oder als ge eines Packaging-Prozesses, der im Allgemeinen Massennomina plu sierbar macht (vgl. (14b) unten und Jackendoff $1991 \mathrm{zu}$ den be Prozessen). In einigen Fällen (vgl. (14c-d) unten) ist eine metaphori bzw. idiombezogene Komponente vorhanden, die weitgehend schwunden bzw. opak geworden sein kann:

(14) a. Der Typ ist echt Banane aber irgentwie [sic!] Geil.

b. Das ist echt eine Scheiße/ ein Schrott/ eine Sabne.

c. Dieser Lehrer ist echt eine Flasche. ${ }^{57}$

d. Dieser Film ist der Hammer: echt sehenswert. 58

Als letzten Typ finden wir eine Reihe von reinen Evaluierungsausdrüc die sich auf ursprüngliche Komposita zurückführen lassen:

57 Vgl. Paul [1897] (2002: 335): ,ugs. übertr. neu ,Versager‘ nach der hohlen leeren Flasc

58 Vgl. Paul [1897] (2002: 448): „vielfältig als Fluch oder Ausruf ..., u.a. nach dem Har des Gottes Thor, noch heute Das ist ein Hammer, das ist großartig' v.a. ugs. (nd. scho Jh. mit abgewandelter Bed.)“. 
Dieser Typ ist relativ neu und wird bei Henzen (1965), aber auch bei 1 scher \& Barz (1992) kaum erwähnt, wenn man von Farbausdrücken lila-, türkisfarben usw. absieht. ${ }^{59}$ Ausdrücke wie bombe und klasse sind ers 19. bzw. 20. Jh. bezeugt, in Komposita wie Bombenerfolg, Bombengedäc bzw. Klasseweib, Klassekäfer, denen eine feste Wendung wie (Weib) Klasse zugrundeliegt (vgl. Paul [1897] 2002: 535) und in Ausdrücken prima Leistung, die ebenfalls auf Wendungen wie Primasorte, von höch Qualität' zurückgehen.

Außerdem genießt dieser Typ heutzutage in gewissen Sprachregis - z.B. der Jugendsprache, vgl. Androutsopoulos (1998) - ein begren sicherlich analogisch gesteuertes Expansionspotential. In dieser Sprac rietät sind nämlich schon seit einiger Zeit solche Verwendungen beob tet worden, wobei „Klasse die zeitlich älteste Konversion [ist] und of sichtlich als Vorbild für das gesamte Modell [diente]“ (Androutsopo 1998: 193).

Bei den letzten Typen, die beide hauptsächlich die Evaluierungsfi tion teilen, wird nun die oben skizzierte Tendenz zur Rückbildung trennbaren Verbkomposita, d. h. von einer die Distanzstellung bzw. 1 tifunktionalität favorisierenden Konstruktion, über den Mechanismus Abkürzung sichtbar. Im Allgemeinen sind nämlich die meisten For dieser zwei letzten Typen auch als Erstglieder in Komposita als Intens rer belegt (vgl. Androutsopoulos 1998: 108):

(16) a. Hammerplatte, Kackplatte, Sabneteil, Scheißtag, Schrotttypen

b. Bombenjob, Höllenlärm, Klassefrau, Kultgruppe, Spitrensound

Offensichtlich ist der Mechanismus der Abkürzung gegenüber dem p lel laufenden Mechanismus der Rückbildung von trennbaren Verbkom sita favorisiert worden, insbesondere bei jenen Komposita, wo der K ein deverbales Abstraktum ist und die Reverbalisierung des Komposit favorisiert:

(17) Klassefrau/Klassespiel

Klassespiel/Klavierspiel

klasse spielen/klavier spielen

59 Vgl. auch Altmann \& Kemmerling (2005: 151): „Schließlich sei noch auf die verbr Konversion bei Farbwörtern hingewiesen: türkis, bordeaux, cognac, flieder, mango, sand, tabac, havanna. Bei attributivem Gebrauch ist die Kombination mit -farben (oft auch notwendig, um Flektierbarkeit zu erreichen. An den Farbbeispielen sieht man, das Prozess synchron aktiv ist“". 
tisch in adverbialer Funktion vorkommt (vgl. (9) oben und Pittne Berman 2006), wobei der zweite Typ ein gemischtes Verhalten aufweis

(18) a. Es ist mir käse.

*Es ist mir käse gegangen.

b. *Es ist mir schrott.

Es ist mir schrott gegangen.

Schwankungen unter den Typen sind sicherlich zu erwarten, wie der panne zeigt. Außerdem ist in manchen Fällen die Zuschreibung zu ei der drei Typen fraglich, wie bei wurst/wurscht, wo es vielleicht nutzlos argumenttragenden prädikativen Ursprung von einer Verwendung askriptives Massennomen zu unterscheiden. Sicherlich ist die idiomati Komponente ziemlich stark. ${ }^{60}$

\section{Fazit}

Aus einer sprachtypologischen Perspektive ist der oben dargestellte $\mathrm{d}$ Typ von hohem Interesse, da über den polysynthetischen Mechanis der Komposition, der im Lauf der deutschen Sprachgeschichte im mehr produktiv geworden ist, mit Hilfe eines Abkürzungsmechanisr der von den naheliegenden Rückbildungen favorisiert bzw. beeinfl wurde, ein nicht unproduktives Muster für die Schaffung von Eval rungsausdrücken entstanden ist, welche hauptsächlich prädikativ adverbial verwendet werden können. Und wie in solchen syntaktisc Stellungen im Deutschen üblich sind diese Evaluierungsausdrücke un tiert, wie schon von Eichinger angedeutet, und erhöhen deswegen Grad an Multifunktionalität in der Sprache. Und dies wiederum ist typisches Merkmal von analytischen Sprachen.

Ein altes Merkmal des Deutschen, das schon in den ältesten Spr stufen seine Spuren hinterlassen hat, wurde also im Zusammenhang der aus der Klammerbildung entstandenen Dekategorialisierung zu ei fruchtbaren Ausdrucksmittel für besondere stilistische Effekte in e Sprachvarietät, die stets auf der Suche nach expressiven, Gruppeniden schaffenden Kodierungspotenzialen ist wie eben die Jugendsprache.

60 Vgl. Röhrich (1992: 1751): „,vielleicht ist nur an die Gleichartigkeit gedacht, die sich b Wurst an beiden Enden zeigt ... Es ist gleichgültig, an welchem Ende die Wurst anges ten wird". 
Admoni, Wladimir (1990), Die Entwicklung des Gestaltungssystems als Grung der historischen Syntax. In: Betten, Anne (Hrsg.), Neuere Forschungen zur histor Syntax des Deutschen. Tübingen: Niemeyer, 1-13.

Aikhenvald, Alexandra Y. (2007), Typological distinctions in word-formation Shopen, Timothy (ed.), Language Typology and Syntactic Description. Vol. 3: Gram cal Categories and the Lexicon. 2. Aufl. Cambridge: Cambridge University Pres 65.

Altmann, Hans \& Silke Kemmerling (2005), Wortbildung fürs Examen, 2. überarb. Göttingen: Vandenhoeck \& Ruprecht.

Androutsopoulos, Jannis K. (1998), Deutsche Jugendsprache. Untersuchungen zu ibren S turen und Funktionen. Frankfurt am Main: Lang.

Booij, Geert (1996), Inherent versus contextual inflection and the split morph hypothesis. In: Booij, Geert \& Jaap van Marle (eds.), Yearbook of Morphology Dordrecht: Kluwer, 1-16.

Booij, Geert (2009), A constructional analysis of quasi-incorporation in Dutch. Kenkyu 135: 5-27.

Dahl, Östen (2004), The Growth and Maintenance of Linguistic Complexity, Amsterda Philadelphia: Benjamins.

Donalies, Elke (2002), Die Wortbildung des Deutschen. Ein Überblick. Tübingen: Narr.

Eichinger, Ludwig M. (2007), Adjektiv und Adkopula. In: Hoffmann, Ludger (H Handbuch der deutschen Wortarten. Berlin \& New York: de Gruyter, 143-187.

Eisenberg, Peter (2004), Grundriss der deutschen Grammatik. Das Wort. 2. überarb. tualisierte Aufl. Stuttgart: Metzler.

Eschenlohr, Stefanie (1999), Vom Nomen zum Verb: Konversion, Präfigierung und Rü dung im Deutschen. Hildesheim: Olms.

Feuillet, Jack (1989), Linguistique diachronique de l'allemand. Bern \& New York: Lang.

Fleischer, Wolfgang \& Irmhild Barz (1992), Wortbildung der deutschen Gegenwartssp Tübingen: Niemeyer.

Fuhrhop, Nanna (2007), Zwischen Wort und Syntagma. Zur grammatischen Fundierun Getrennt- und Zusammenschreibung. Tübingen: Niemeyer.

Gaeta, Livio (2007), On the double nature of productivity in inflectional morpho Morphology 17: 181-205.

Gaeta, Livio (2009), Inflectional morphology and productivity: Considering qualit and quantitative approaches. In: Krifka, Manfred \& Patrick Steinkrüger (eds. Inflection. Berlin \& New York: Mouton de Gruyter, 45-68.

Gaeta, Livio (im Druck), Synthetic compounds: with special reference to Germat Scalise, Sergio \& Irene Vogel (eds.), Compounding. Amsterdam \& Philadel Benjamins.

Gaeta, Livio \& Davide Ricca (im Druck), Composita solvantur: Compounds as le units or morphological objects?. In: Gaeta, Livio \& Maria Grossmann ( Compounds between syntax and lexicon. Sonderheft von Italian Journal of Lingui Rivista di Linguistica 21.1.

Henzen, Walter (1965), Deutsche Wortbildung. 3. durchges. u. ergänzte Aufl. Tübir Niemeyer.

Hohenhaus Peter (2005), Lexicalization and institutionalization. In: Štekauer, Pav Rochelle Lieber (eds.). Handbook of Word-Formation. Dordrecht: Springer, 353 
Iturrioz Leza, José Luıs (2001), Inkorporation. In: Haspelmath, Martın, Ekke König, Wulf Oesterreicher \& Wolfgang Raible (eds.), Language Typology and L age Universals. 1. Halbband. Berlin \& New York: de Gruyter, 714-725.

Jackendoff, Ray (1991), Parts and boundaries. Cognition 41: 9-45.

Kühnhold, Ingeburg, Oskar Putzer \& Hans Wellmann (1978), Deutsche Wortbi Typen und Tendenzen in der Gegenwartssprache. Bd. 3: Das Adjektiv. Düssel Schwann.

Lockwood, William B. (1968), Historical German syntax. Oxford: Clarendon.

Motsch, Wolfgang (2004), Deutsche Wortbildung in Grundzügen. 2. Aufl. Berlin \& York: Mouton de Gruyter.

Nübling, Damaris (2005), Von in die über in'n und ins bis im: Die Klitisierung Präposition und Artikel als „Grammatikalisierungsbaustelle“. In: Leusc Torsten, Tanja Mortelmans \& Sarah De Groodt (Hrsg.), Grammatikalisieru Deutschen. Berlin \& New York: de Gruyter, 105-131.

Nübling, Damaris (2006), Historische Sprachwissenschaft des Deutschen: eine Einfübrung Prinzipien des Sprachwandels. (In Zusammenarbeit mit Antje Dammel, Janet I und Renata Szczepaniak). Tübingen: Narr.

Paul, Hermann [1897] (2002), Deutsches Wörterbuch. 10. überarb. und erw. Aufl. Helmut Henne, Heidrun Kämper \& Georg Objartel. Tübingen: Niemeyer.

Pittner, Karin \& Judith Berman (2006), video ist echt schrott aber single ist hammer. Jug sprachliche Nomen-Adjektiv-Konversion in der Prädikativposition. Deutsche che 34: 233-250.

Primus, Beatrice (1997), Der Wortgruppenaufbau in der Geschichte des Deuts Zur Präzisierung von synthetisch vs. analytisch. Spracbwissenschaft 22: 133-159.

Roelcke, Thorsten (2004), Analytismus im Deutschen. In: Hinrichs, Uwe (Hrsg.) europäischen Sprachen auf dem Wege zum analytischen Sprachtyp. Wiesbaden: Har witz, 147-168.

Röhrich, Lutz (1992), Das große Lexikon der sprichwörtlichen Redensarten. Freibur Breisgau: Herder.

Ronneberger-Sibold, Elke (1994), Konservative Nominalflexion und „klammer Verfahren“ im Deutschen. In: Köpcke, Klaus-Michael (Hrsg.), Untersuchunge deutschen Nominal- und Verbalmorphologie. Tübingen: Niemeyer, 115-130.

Skalička, Vladimír (1979), Typologische Studien. Braunschweig: Viehweg.

Thurmair, Maria (1991), Warten auf das Verb. Die Gedächtnisrelevanz der klammer im Deutschen. Jabrbuch Deutsch als Fremdsprache 17: 174-202.

Vogel, Petra M. (1996), Wortarten und Wortartenwechsel. Zu Konversion und verwa Erscheinungen im Deutschen und in anderen Sprachen. Berlin \& New York: de Gruy

Vogel, Petra M. (1997), Unflektierte Adjektive im Deutschen: Zum Verhältnis semantischer Struktur und syntaktischer Funktion und ein Vergleich mit and Adjektiven. Sprachwissenschaft 22: 403-433.

Wegener, Heide (1999), Zum Bedeutungs- und Konstruktionswandel bei psychis Verben. In: Wegener, Heide (Hrsg.), Deutsch kontrastiv. Typologisch-vergleichende tersuchungen zur deutschen Grammatik. Tübingen: Stauffenburg, 171-210.

Weinrich, Harald (2005), Textgrammatik der deutschen Sprache. 3. revid. Aufl. Hildesh Olms. 
dam \& Philadelphia: Benjamıns, 959-988.

Wurzel, Wolfgang U. (1996a), Morphologischer Strukturwandel: Typologische wicklungen im Deutschen. In: Lang, Ewald \& Gisela Zifonun (Hrsg.), Deut typologisch. Berlin \& New York: de Gruyter, 492-524.

Wurzel, Wolfgang U. (1996b), On the similarities and differences between inflect and derivational morphology. Sprachtypologie und Universalienforschung 49.3: 267-

Wurzel, Wolfgang U. (1998), On the development of incorporating structur German. In: Hogg, Richard M. \& Linda van Bergen (eds.), Historical Ling 1995. Volume 2: Germanic Linguistics. Amsterdam \& Philadelphia: Benjamins, 344.

Zifonun, Gisela, Ludger Hoffmann \& Bruno Strecker (1997), Grammatik der den Sprache. (3 Bände). Berlin \& New York: de Gruyter. 
\& Barz 1992; D = Donalies 2002; M = Motsch 2004; AK = Altmann \& K merling 2005; A = Androutsopoulos 1998; PB = Pittner \& Berman 2006.

\begin{tabular}{|c|c|c|c|c|c|c|c|}
\hline & $\mathrm{H}$ & KPW & FB & D & M & $\mathrm{AK}$ & $\mathrm{A}+$ \\
\hline abrede & + & & & & & & \\
\hline angst & + & + & + & + & & + & \\
\hline \multicolumn{8}{|l|}{ banane } \\
\hline \multicolumn{8}{|l|}{ bang(e) } \\
\hline bankrott & + & & & & & & \\
\hline barock & & & + & & + & + & \\
\hline bordeaux & & & + & & & + & \\
\hline brach & + & & & & & & \\
\hline cognac & & & + & & & + & \\
\hline dunkel & & & & & + & & \\
\hline durft & + & & & & & & \\
\hline ekel & + & & & + & & & \\
\hline elend & & & & & + & & \\
\hline ernst & + & + & + & + & + & + & \\
\hline esel & + & & & & & & \\
\hline fehl & + & & & & & & \\
\hline feind & + & + & + & + & & + & \\
\hline \multicolumn{8}{|l|}{ flasche } \\
\hline flieder & & & + & & & + & \\
\hline \multicolumn{8}{|l|}{ flop } \\
\hline freund & & + & + & + & & & \\
\hline fromm & + & & & & & & \\
\hline gewalt & + & & & & & & \\
\hline giwar & + & & & & & & \\
\hline giwon & + & & & & & & \\
\hline \multicolumn{8}{|l|}{ Gott } \\
\hline \multicolumn{8}{|l|}{ gram } \\
\hline grimm & & & & + & & & \\
\hline \multicolumn{8}{|l|}{ hammer } \\
\hline harm & + & & & & & & \\
\hline havanna & & & + & & & + & \\
\hline \multicolumn{8}{|l|}{ hölle } \\
\hline indigo & & & + & & & & \\
\hline \multicolumn{8}{|l|}{ kacke } \\
\hline \multicolumn{8}{|l|}{ kaputt } \\
\hline \multicolumn{8}{|l|}{ käse } \\
\hline klasse & & + & + & & + & + & \\
\hline knaller & & & & & & & \\
\hline
\end{tabular}




\begin{tabular}{|c|c|c|c|c|c|c|c|}
\hline knüller & & & & & & & \\
\hline koralle & & & + & & & & \\
\hline \multicolumn{8}{|l|}{ kult } \\
\hline leid & & & & + & & & \\
\hline lila & + & & & & & & \\
\hline lind & & & + & & & & \\
\hline mango & & & + & & & & \\
\hline \multicolumn{8}{|l|}{ mist } \\
\hline \multicolumn{8}{|l|}{ müll } \\
\hline not & + & & + & + & & + & \\
\hline \multicolumn{8}{|l|}{ orange } \\
\hline \multicolumn{8}{|l|}{ panne } \\
\hline \multicolumn{8}{|l|}{ piep } \\
\hline pleite & & & & + & & & \\
\hline revolutionär & & & + & & & + & \\
\hline rosa & + & & & & & & \\
\hline \multicolumn{8}{|l|}{ sahne } \\
\hline sand & & & + & & & + & \\
\hline schachmat & + & & & & & & \\
\hline schade & + & & & & & & \\
\hline \multicolumn{8}{|l|}{ scheiße } \\
\hline schilf & & & + & & & + & \\
\hline schmuck & + & + & + & + & + & + & \\
\hline schnuppe & + & & & & & & \\
\hline \multicolumn{8}{|l|}{ schnurz } \\
\hline \multicolumn{8}{|l|}{ schrott } \\
\hline schuld & + & + & + & + & & + & \\
\hline \multicolumn{8}{|l|}{ spitze } \\
\hline tabac & & & + & & & + & \\
\hline teig & + & & & & & & \\
\hline \multicolumn{8}{|l|}{ toto } \\
\hline türkis & & & & & & + & \\
\hline vanille & & & + & & & & \\
\hline wenig & + & & & & & & \\
\hline wette & + & & & & & & \\
\hline wrac & + & & & & & & \\
\hline wurst & + & & & & & & \\
\hline \multicolumn{8}{|l|}{ zimt } \\
\hline zwanzig & + & & & & & & \\
\hline
\end{tabular}


Brought to you by | Interscientia S.A.S. (Universita Studi di Torino Biblioteca Ruffini Authenticated | 172.16.1.226

Download Date | 4/2/12 7:16 PM 